Cascales-Martínez, A. \& Gomariz-Vicente, M.A. (2021). Acción Tutorial en Formación Profesional: perspectiva del profesorado. Revista Electrónica Interuniversitaria de Formación del Profesorado, 24(2), 51-65.

DOI: https://doi.org/10.6018/reifop.453461

\title{
Acción Tutorial en Formación Profesional: perspectiva del profesorado
}

Antonia Cascales-Martínez, Mª́ngeles Gomariz Vicente

Universidad de Murcia

\section{Resumen}

La Acción Tutorial (AT) es una labor integrada en todos los niveles del sistema educativo, y por tanto en Formación Profesional (FP). A través de ella, los docentes favorecen las relaciones que se establecen en el aula con los distintos agentes implicados. El objetivo es conocer la opinión de los docentes que imparten en FP de la Región de Murcia sobre el desarrollo de la AT. Para ello se ha seguido un método no experimental, cuantitativo, tipo encuesta, con una muestra de 60 docentes. Para la recogida de datos se ha empleado el cuestionario generado tras el proceso de validación. Los resultados obtenidos destacan el beneficio de la tutoría dentro de las relaciones en el aula en FP, a la vez que consideran contribuye a mejorar diferentes aspectos en la evolución de los alumnos a nivel académico, profesional y personal, si bien necesitan de más tiempo y formación.

\section{Palabras clave}

Formación Profesional; Enseñanza Tutorial; orientación; tutor.

\section{Tutorial Action in Vocational Training: perspective of the teaching staff}

\section{Abstract}

Tutorial Action (AT) is an integrated task at all levels of the educational system, and therefore in Vocational Training (FP). Through it, teachers promote relationships established in the classroom with the different agents involved. The objective is to know the opinion of the teachers who teach in FP of the Region of Murcia on the development of the AT. For this, a 
non-experimental, quantitative survey-type method was followed, with a sample of 60 teachers. For data collection, the questionnaire generated after the validation process has been used. The results obtained highlight the benefit of tutoring within classroom relationships in VET, while considering that it contributes to improving different aspects of the evolution of students at an academic, professional and personal level, although they need more time and training.

\section{Key words}

Vocational Training; Tutorial Action; orientation; tutor

\section{Introducción}

La práctica de la Acción Tutorial (AT) se considera como un indicador de calidad en educación, y se plantea como una actividad que debe estar presente en todas las etapas educativas, incluida la Formación Profesional. Todos los docentes, incluidos aquellos que imparten docencia en los ciclos formativos de Formación Profesional, tienen que llevar a cabo una función tutorial que está implícita en su propia actividad docente como elemento inherente a ella (Castillo et al., 2009) y con el objetivo de lograr la educación integral del alumnado, fin último de la educación, que implica una capacitación pedagógica con la que los docentes pueden no contar. Por tanto, dada la importancia de la función tutorial en la Formación Profesional nos induce a entender que se debe profundizar en su estudio (González, 2018) a fin de determinar cuales son las necesidades de este colectivo tan importante para la sociedad.

La AT es un proceso complejo ya que está íntimamente ligado a la interacción humana, por eso es un requisito imprescindible que el tutor se forme para lograr la formación integral de los alumnos (García et al., 2016). La AT debe ser colectiva y coordinada por parte de todo el profesorado que imparte docencia a un grupo de alumnos, lo cual supone, que debe realizarse dentro de un nuevo enfoque más comprensivo e integral de la tutoría (Álvarez, 2017). Representa la faceta más humana de la docencia, entre sus objetivos podemos distinguir el conocimiento y la valoración de los alumnos, para así ser capaz de orientarlos desde el principio de la individualidad, promover la socialización del grupo clase con la intención de favorecer la cohesión del grupo, detectar y llevar a cabo las adaptaciones curriculares pertinentes atendiendo a la diversidad del alumnado (García et al., 2016; Sánchez, Campillo, De Nicolás y Peral, 2016).

Como ya hemos indicado, la tutoría es el primer nivel de la orientación educativa, siendo el segundo y el tercer nivel, el Departamento de Orientación y los equipos de orientación educativa y psicopedagógica, respectivamente (Sánchez et al, 2016). Todos comparten una finalidad orientadora y deben trabajar de forma cooperativa (Caja, Cascales y Gomáriz, 2017; Narro y Arredondo, 2013; Torres, 1996), ello sustenta en el hecho de que el aprendizaje es sumamente complejo dado que intervienen todas las dimensiones de la persona. El concepto de tutoría va ligado a los conceptos de educación y orientación, y en ese sentido, entendemos que la tutoría se puede considerar como un proceso de guía (Lázaro, 1997) y acompañamiento del alumnado (Álvarez, 2020; Castillo, Torres y Polanco, 2009) que está integrado actualmente en todos los niveles del sistema educativo, incluida la Formación Profesional. Otros autores señalan la tutoría como un proceso continuo, no puntual, planificado y sistemático, activo y dinámico e interdisciplinar. Por ello, trata de dirigir el progreso del alumnado en sus distintas facetas, académicas, personales o profesionales, de manera que se propicie el desarrollo integral de los alumnos (Álvarez, 2017). 
La función tutorial es responsabilidad de toda la comunidad educativa, por tanto, todo profesor debe asumir una labor orientadora que se lleva a cabo mediante la AT y que cuenta con la figura del tutor de clase como coordinador (Torrego et al., 2014). Ello queda recogido en las funciones del profesorado establecidas en la Ley Orgánica de Educación (LOE, 2006), que han sido mantenidas por la Ley Orgánica de Mejora de la Calidad en Educación (LOMCE, 2013), refiriéndose a aspectos vinculados a la AT de la que se hace responsable a todo el profesorado, sin distinción de rol ni etapa educativa.

El Reglamento Orgánico de Institutos de Educación Secundaria (ROI-ES), aprobado por el Real Decreto 83/1996, de 26 de enero, establece que cada grupo de alumnos, tendrá un tutor designado por el director del Centro, a propuesta del jefe de estudios, el cual tendrá asignadas unas funciones que aparecen reflejadas en el artículo 56.1 del ROI-ES y en el artículo 56.2 del ROI-ES se concretan las funciones en el caso de la Formación Profesional. Ante esta normativa, Arnaiz y Riart (1999) señalan que el tutor es el orientador más próximo al grupo y debe ser una persona capacitada para esta función.

Respecto a la capacitación del profesorado, para impartir enseñanzas de Formación Profesional, según establece la LOE (2006), se exige tener una formación pedagógica y didáctica a nivel de Postgrado. En este contexto, adquiere sentido la afirmación de Álvarez (2017) cuando señala que todo docente está implicado en la AT, independientemente de que se le asigne un grupo de alumnos como tutor, dado que cuenta con una formación inicial. Otros autores consideran que el docente necesita una formación más especifica para el desarrollo de competencias relacionadas con la AT dada la importancia para lograr sesiones eficaces durante las tutorías con los alumnos (Álvarez, 2019). Dicha formación debe incidir en la capacidad del tutor para establecer relaciones e interacciones personales con el alumnado, familia y docentes, desarrollando un sentimiento de pertenencia al grupo. Otros autores, añaden como competencias las que abarca la tutoría la comunicación eficaz, la escucha activa, el manejo de conflictos, la participación, el trabajo en equipo y la planificación del tiempo, todo ello guiado por principios éticos (Castaño, Blanco y Asensio, 2012; Tárrida, 2012).

Para que la AT se desarrolle de manera planificada y satisfactoria de acuerdo con sus necesidades hay que diseñar el Plan de Acción Tutorial (PAT), definido como la respuesta que, de un modo sistemático e intencional, realiza el centro educativo para concretar operativamente la concepción de la orientación educativa que quiere desarrollar a favor de sus estudiantes (Castillo et al., 2009). Se convierte en una herramienta que facilita el ajuste de las actividades planificadas a los objetivos, a las prioridades y al contexto del ámbito de actuación.

Es la Resolución de 3 de septiembre de 2003, de la Dirección General de Enseñanzas Escolares, por la que se dictan instrucciones para el funcionamiento de los departamentos de orientación en los centros de Educación Secundaria sostenidos con fondos públicos de la Comunidad Autónoma de la Región de Murcia (CARM), la que indica que el Departamento de Orientación se encarga de elaborar el PAT en colaboración con los tutores y siguiendo las directrices establecidas por la comisión pedagógica. La resolución de 9 de abril de 2015 de la Dirección General de Formación Profesional y Enseñanzas de Régimen Especial por la que se dictan las instrucciones para la puesta en marcha y desarrollo del módulo de Formación en Centros de Trabajo en centros docentes de la CARM, dice que la tutoría será considerada parte integrante de este módulo, y que cada 15 días se realizará una sesión de tutoría, que se desarrollará en el centro formativo. El tiempo destinado a este tipo de tutorías no superarán el $10 \%$ del total de las horas destinadas al desarrollo de este módulo. Por otra parte, la resolución de 8 de noviembre de 2018 de la Dirección General de Formación Profesional y Enseñanzas de Régimen Especial por la que se dictan instrucciones para el desarrollo del módulo profesional de Proyecto de los ciclos formativos de FP en el ámbito de la CARM, 
señala que la planificación de este módulo debe incluir la planificación de las tutorías y da cabida tanto a la tutoría individual como a la colectiva. Las sesiones de tutoría individual, en el módulo de Proyecto, se distribuyen en 2 horas quincenales e irán destinadas a la resolución de dudas. La tutoría colectiva, con carácter inicial, irá destinada a familiarizar al alumnado con el método de trabajo del proyecto y a concretar los aspectos formales que debe contener un proyecto.

Entre las dificultades para la integración de la AT en el aula, encontramos la falta de tiempo para llevarla a cabo, la falta de interés por parte del alumnado o el escaso compromiso por parte del profesorado por asumir el rol de tutor (Álvarez y Álvarez, 2015).

Para la formación integral del alumnado, es necesario que la AT abarque toda la etapa educativa de la Formación Profesional, si bien es cierto, que la AT debe estar integrada dentro de las programaciones de cada uno de los módulos, y al no existir un horario lectivo, corre el peligro de ser dejada de lado debido a la falta de tiempo, o a la falta de interés por parte de alumnos o profesores-tutores.

Desde la AT se pueden crear unas condiciones que favorezcan la convivencia y la cooperación en el aula a la vez que mejoren las relaciones profesor-alumno, algo que influye en el éxito de los alumnos y en la convivencia dentro del aula y del centro.

La AT contribuirá a un mejor desarrollo personal y profesional del grupo de alumnos si facilita que el alumnado asuma valores, normas y hábitos de trabajo, además los datos sugieren que desde la AT se puede fomentar la responsabilidad y autonomía de trabajo y la resolución conjunta de situaciones conflictivas, lo que va a provocar un clima positivo (Olmos Rueda y Mas Torelló, 2017; Serrano, 2009).

Por otra parte, teniendo en cuenta la individualidad del alumnado, la AT es un punto de apoyo que les ofrece información y orientación sobre su propia formación, incidiendo de manera positiva en su proceso de aprendizaje, contribuyendo al desarrollo de sus competencias académicas y profesionales, y guiándolo en la elaboración y desarrollo de su propio proyecto personal (Castaño et al., 2012). Además, el profesor puede aprovechar para recoger información del alumnado que mejore su docencia y así modificar las prioridades y los métodos de trabajo de acuerdo a dinámicas de trabajo más adaptadas al alumnado atendiendo a la diversidad (López y Cascales, 2019; Álvarez, 2008).

En el caso que nos ocupa, que es el ámbito de la formación profesional, la AT deber ir destinada, además, a favor de la integración y adaptación del alumnado al mundo laboral, ya que el propósito de este tipo de formación va dirigido a preparar a los alumnos para el desempeño de un oficio o profesión, y por tanto, para que la incorporación del alumnado al mundo laboral se lleve a cabo con éxito en un contexto donde la realidad laboral está en continuo cambio. El enfoque hacia la orientación profesional del alumnado en los ciclos formativos adquiere una mayor importancia en los módulos de Formación y Orientación Laboral y en Formación en centros de trabajo (Olmos Rueda y Mas Torelló, 2017).

Para ellos es necesario tener un conocimiento previo del alumnado, sus metas profesionales $y$, por supuesto, su procedencia académica. Dado que nos encontramos os encontramos ante grupos de alumnos que presentan unas características muy heterogéneas en cuanto a su edad, conocimientos previos y metas profesionales, lo que condicionará su propia motivación, y esto debe ser tenido en cuenta por parte de los profesores en el ejercicio de la docencia y en especial en el de la AT a la hora de considerar a los alumnos como individuos y como grupo y de adaptar el PAT a las características concretas del contexto en el que se desarrolla su docencia. 
En este sentido, el objetivo general de esta investigación es conocer la opinión de los docentes que imparten en Formación Profesional de la Región de Murcia sobre el desarrollo de la AT en esta etapa educativa. Esto se concreta en los siguientes objetivos específicos:

1. Averiguar si la AT se integra en las aulas de Formación Profesional, desde la perspectiva de los docentes de esta etapa.

2. Estimar la relación entre los docentes, el Departamento de Orientación y el Plan de Acción Tutorial, en opinión del profesorado de Formación Profesional.

3. Determinar si la AT contribuye a mejorar las relaciones en el aula de Formación Profesional, según la percepción del profesorado de FP.

4. Valorar la importancia del desarrollo profesional del alumno en la AT en Formación Profesional.

5. Conocer la satisfacción del profesorado de Formación Profesional respecto al ejercicio de la AT.

\section{Método}

El diseño empleado para la investigación fue no experimental, de carácter cuantitativo, transversal, no experimental, descriptivo, tipo encuesta (Bisquerra, 2009). Este tipo de método nos permite aplicar el método científico para comprender, conocer y explicar la realidad educativa, con el objeto de desarrollar el conocimiento científico de la educación.

La muestra está compuesta por 60 docentes que imparten Formación Profesional en la Región de Murcia pertenecientes a 10 familias profesionales diferentes. Para la selección se empleó un muestreo no aleatorio por conveniencia. La descripción de las características de los participantes queda recogida en la Tabla 1.

Tabla 1

Variables demográficas de la muestra

\begin{tabular}{|c|c|c|c|}
\hline Variables & Profesorado & $\%$ & Frecuencia \\
\hline \multirow[t]{2}{*}{ Sexo } & Hombres & $53.3 \%$ & 32 \\
\hline & Mujeres & $46.7 \%$ & 28 \\
\hline \multirow[t]{2}{*}{ Titularidad centro } & Centros públicos & $83.3 \%$ & 50 \\
\hline & Centros concertados & $16.7 \%$ & 10 \\
\hline \multirow[t]{3}{*}{ Edad } & Menores de 30 años & $3.3 \%$ & 2 \\
\hline & Entre $30-60$ años & $93.3 \%$ & 56 \\
\hline & Mayores de 60 años & $3 \cdot 3 \%$ & 2 \\
\hline \multirow[t]{2}{*}{ Tutoría } & Tutores & $66.7 \%$ & 40 \\
\hline & No tutores & $33.3 \%$ & 20 \\
\hline \multirow{3}{*}{$\begin{array}{l}\text { Experiencia } \\
\text { docente }\end{array}$} & Menos de 5 años & $21.7 \%$ & 13 \\
\hline & Entre 5 y 20 años & $46.7 \%$ & 28 \\
\hline & Más de 20 años & $31.7 \%$ & 19 \\
\hline \multirow{2}{*}{$\begin{array}{l}\text { Formación en } \\
\text { Acción Tutorial }\end{array}$} & Con formación & $58.3 \%$ & 35 \\
\hline & Sin formación & $41.7 \%$ & 25 \\
\hline
\end{tabular}




\section{Instrumento}

Se creó una encuesta ad hoc (Cerón Gómez, Cascales Martínez y Gomariz Vicente, 2019) que incluye dos bloques; un primer bloque de datos sociodemográficos y un segundo bloque donde se recoge el cuestionario general que consta de 26 ítems y que está basado en una escala tipo Likert con cinco opciones de respuesta donde 1 es Nunca y 5 Siempre. En la Figura 1 podemos ver las dimensiones que incluye el cuestionario:
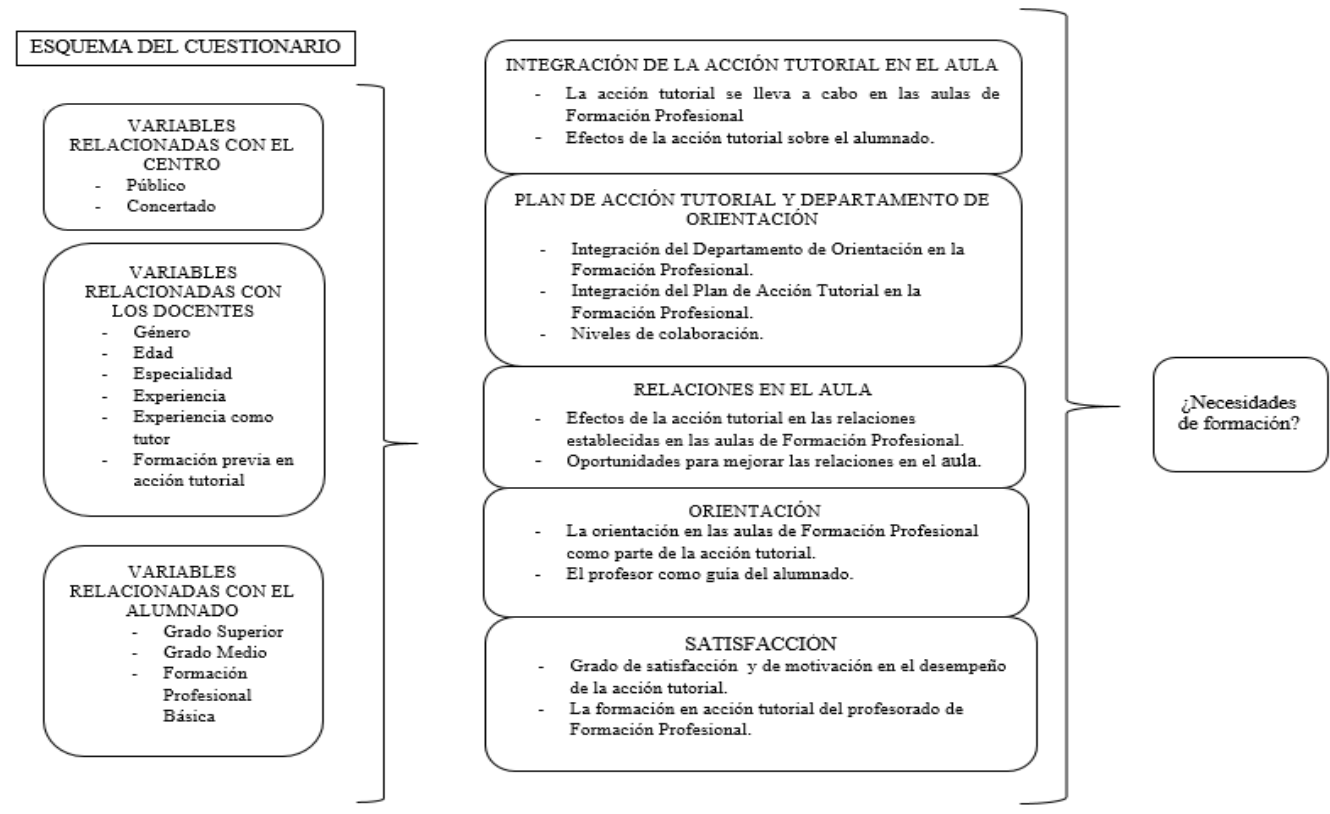

Figura 1. Esquema con dimensiones del cuestionario ad hoc.

La validez de contenido del cuestionario quedó salvaguardada por la participación de un grupo de expertos, quienes participaron en la validación a través de juicio de expertos del instrumento, atendiendo fundamentalmente a su claridad y relevancia. En cuanto a la fiabilidad, arrojó un coeficiente alpha de Cronbach de .898, lo que indica una elevada consistencia interna (De Vellis, 2003). Al someter de manera independiente las distintas dimensiones de nuestro cuestionario a la prueba del alfa de Cronbach, encontramos los resultados de confiabilidad bueno y muy bueno.

\section{Análisis de datos}

El análisis de datos se llevó a cabo con el programa SPSS Versión 24, herramienta que nos permitió hacer un análisis del coeficiente de fiabilidad Alfa de Cronbach especificado anteriormente, un análisis descriptivo (frecuencias, porcentajes, medias y desviaciones típicas) y un análisis inferencial usando técnicas de estadística no paramétrica de los datos recogidos con nuestro cuestionario. Además, se calculó el tamaño del efecto a través del estadístico d de Cohen (Cohen, 1988).

\section{Resultados}

El resumen de los resultados obtenidos de acuerdo con los objetivos planteados.

Objetivo 1: Averiguar si la AT se integra en las aulas de Formación Profesional, desde la perspectiva de los docentes de esta etapa. 
En la Tabla 2 podemos apreciar de manera general que, en opinión de los docentes de FP participantes, la integración de la AT en el aula es buena, ya que hemos obtenido una media en el total de la dimensión de 3.73 .

Respecto a los beneficios de la AT en el rendimiento académico más del $80 \%$ de los encuestados está de acuerdo en que siempre o casi siempre es positiva, los profesores que no son tutores y los que no han recibido formación en AT dan respuestas más positivas en este ítem, con una significación estadística del 0.034 y 0.031 respectivamente.

Igualmente, para los beneficios de la AT en el desarrollo personal de los alumnos, más del $80 \%$ piensa que siempre o casi siempre son favorables y los profesores que no tienen formación en AT dan respuestas significativamente más positivas, con una significación estadística de $p=.001$ y con un tamaño del efecto $d$ de Cohen intenso, pues es superior a .50 .

Sin embargo, contrastando con los resultados obtenidos en el resto de ítems que componen esta dimensión, en el caso del tiempo destinado a la Acción Tutorial, el $41.7 \%$ de los encuestados afirma que las horas destinadas a esta labor nunca son suficientes.

Tabla 2.

Estadísticos descriptivos de la dimensión Integración de la AT en el aula

\begin{tabular}{|c|c|c|c|c|c|c|c|c|}
\hline \multicolumn{9}{|c|}{ Porcentajes } \\
\hline Ítem & Media & Mediana & $\begin{array}{l}\text { Desv. } \\
\text { Típica }\end{array}$ & Nunca & $\begin{array}{l}\text { Casi } \\
\text { Nunca }\end{array}$ & $\begin{array}{l}A \\
\text { veces }\end{array}$ & $\begin{array}{l}\text { Casi } \\
\text { siempre }\end{array}$ & Siempre \\
\hline $\begin{array}{l}\text { 1. La AT está integrada } \\
\text { en la acción docente } \\
\text { diaria }\end{array}$ & 3.97 & 4.0 & 1.025 & 1.7 & 6.7 & 23.3 & 30 & 38.3 \\
\hline $\begin{array}{l}\text { 2. La AT se desarrolla } \\
\text { en el aula de forma } \\
\text { activa y dinámica }\end{array}$ & 3.92 & 4.0 & .944 & 1.7 & 3.3 & 28.3 & 35.0 & 31.7 \\
\hline $\begin{array}{l}\text { 3. La AT contribuye a } \\
\text { mejorar } \\
\text { rendimiento } \\
\begin{array}{ll}\text { académico de los } \\
\text { alumnos }\end{array} \\
\end{array}$ & 4.92 & 4.0 & .804 & - & $3 \cdot 3$ & $13 \cdot 3$ & 41.7 & 41.7 \\
\hline $\begin{array}{l}\text { 4. La AT contribuye a } \\
\text { mejorar el desarrollo } \\
\text { personal de los } \\
\text { alumnos }\end{array}$ & 4.28 & 5.0 & .94 & 1.7 & 3.3 & $13 \cdot 3$ & 28.3 & 53.3 \\
\hline $\begin{array}{l}5 . \quad \text { Las horas } \\
\text { destinadas a la AT en } \\
\text { el aula son suficientes }\end{array}$ & 2.28 & 2.0 & 1.303 & 41.7 & $13 \cdot 3$ & 26.7 & 11.7 & 6.7 \\
\hline Total & 3.73 & 3.80 & .650 & & & & & \\
\hline
\end{tabular}


Objetivo 2: Estimar la relación entre el Plan de Acción Tutorial, el Departamento de Orientación y los docentes, en opinión del profesorado de FP.

Según los resultados recogidos en la Tabla 3, la valoración que el profesorado encuestado tiene respecto a las tareas que tienen relación con el PAT y el Departamento de Orientación del centro es positiva como nos muestra la media total de esta dimensión, que se sitúa en 3.61.

Cuando preguntamos por el Plan de Acción Tutorial, el 65\% de los encuestados dice que siempre o casi siempre lo conoce, y las docentes mujeres dan respuestas más positivas, de manera estadísticamente significativa, en cuanto a este aspecto con un nivel de significación de $p=.041$, con un tamaño el efecto de Cohen relevante, superior a .50 .

Por otra parte, también está en torno al $40 \%$ el profesorado participante que se coordina y pide asesoramiento siempre o casi siempre al Departamento de Orientación. Las docentes mujeres, con un nivel de significación de $p=.02$, muestran respuestas significativamente más positivas a la hora de pedir asesoramiento a este departamento, con un valor $\mathrm{d}$ de Cohen considerado relevante, superior a .50 .

Tabla 3.

Estadísticos descriptivos de la dimensión PAT y Departamento de Orientación

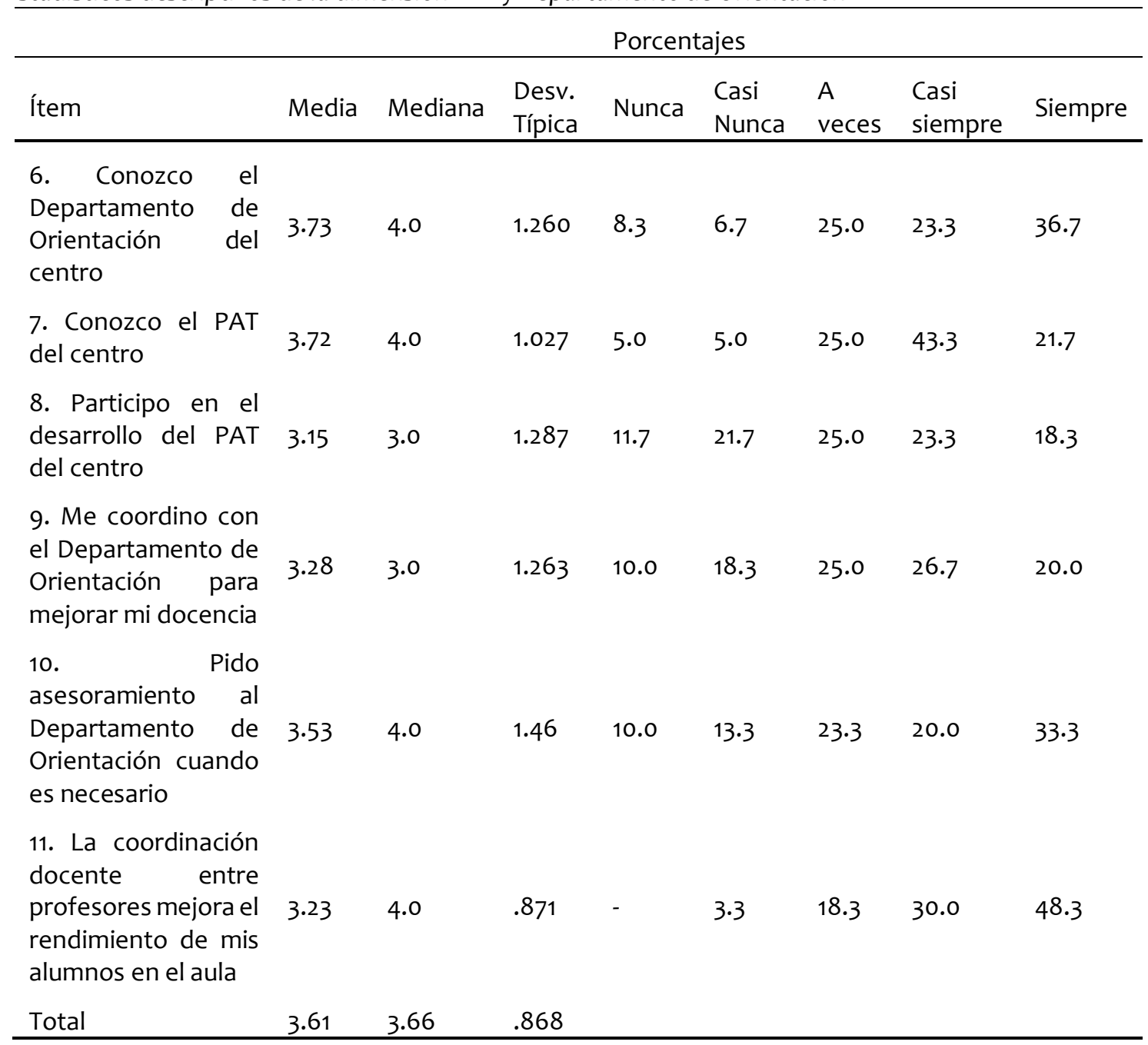


Objetivo 3: Determinar si la AT contribuye a mejorar las relaciones en el aula de Formación Profesional, según la percepción del profesorado de FP.

En la Tabla 4 podemos apreciar, de manera general, que esta dimensión obtiene una media total de 4.34 , lo que indica que los profesores encuestados valoran la AT como un recurso positivo para mejorar las relaciones en el aula.

El 51.7\% de los docentes participantes opina que siempre actúa como mediador entre los alumnos y el centro, el $65 \%$ que siempre aprovecha las oportunidades para favorecer la socialización del grupo y el $65 \%$ que la AT contribuye a mejorar las relaciones en el aula, en los tres casos ningún profesor respondió que nunca lo hace, además, fueron las mujeres las que dieron respuestas significativamente más positivas, con un nivel de significación de $p=.025$, $.010, .025$, respectivamente, con un tamaño del efecto $d$ de Cohen superior a .50 . En el caso de si la AT contribuye a mejorar las relaciones en el aula, los profesores que no tienen formación en Acción Tutorial, también dan respuestas significativamente más positivas con una significación estadística de $p=.014$ y un tamaño del efecto de Cohen superior a .50.

Tabla 4.

Estadísticos descriptivos de la dimensión Relaciones en el aula

\begin{tabular}{|c|c|c|c|c|c|c|c|c|}
\hline \multirow[b]{2}{*}{ Ítem } & \multicolumn{8}{|c|}{ Porcentajes } \\
\hline & Media & Mediana & $\begin{array}{l}\text { Desv. } \\
\text { Típica }\end{array}$ & Nunca & $\begin{array}{l}\text { Casi } \\
\text { Nunca }\end{array}$ & $\begin{array}{l}A \\
\text { veces }\end{array}$ & $\begin{array}{l}\text { Casi } \\
\text { siempre }\end{array}$ & Siempre \\
\hline $\begin{array}{l}\text { 12. Recojo información de } \\
\text { cada uno de mis alumnos } \\
\text { en su registro personal }\end{array}$ & 4.07 & 4.0 & 1.039 & 1.7 & 10.0 & 10.0 & 36.7 & 41.7 \\
\hline $\begin{array}{l}\text { 13. Mis alumnos me } \\
\text { transmiten } \\
\text { inquietudes y problemas }\end{array}$ & 4.15 & 4.0 & $\cdot 917$ & 1.7 & 1.7 & 20.0 & 33.3 & 43.3 \\
\hline $\begin{array}{l}\text { 14. Actúo como mediador } \\
\text { entre mis alumnos y el } \\
\text { centro }\end{array}$ & 4.40 & 5.0 & .718 & - & 1.7 & 8.3 & 38.3 & 51.7 \\
\hline $\begin{array}{l}\text { 15. Aprovecho las } \\
\text { oportunidades para } \\
\text { favorecer la socialización } \\
\text { del grupo }\end{array}$ & 4.53 & 5.0 & .747 & - & 3.3 & 5.0 & 26.7 & 65.0 \\
\hline $\begin{array}{l}\text { 16. La AT contribuye a } \\
\text { mejorar las relaciones en el } \\
\text { aula }\end{array}$ & 4.53 & 5.0 & .747 & - & $3 \cdot 3$ & 5.0 & 26.7 & 65.0 \\
\hline Total & 4.34 & 4.60 & .634 & & & & & \\
\hline
\end{tabular}

Objetivo 4: Valorar la importancia del desarrollo profesional del alumno en la AT en Formación Profesional.

De acuerdo con los datos recogidos en la Tabla 5 el análisis de esta dimensión nos indica un compromiso muy alto por parte de los profesores encuestados respecto a la orientación profesional de los alumnos, ya que la media total de la dimensión es de 4.53, y podemos destacar que ningún profesor da la respuesta nunca en los ítems que componen esta dimensión. 
En lo referente al interés de los profesores por conocer los intereses profesionales de sus alumnos, un 70\% afirma que lo hace siempre y las mujeres se muestran más dispuestas en este aspecto, de manera estadísticamente significativa, con un nivel de significación de $p=.012$ y un tamaño del efecto $d$ de Cohen superior a .50 .

Tabla 5.

Estadísticos descriptivos de la dimensión Orientación profesional

\begin{tabular}{|c|c|c|c|c|c|c|c|c|}
\hline \multirow[b]{2}{*}{ Ítem } & \multicolumn{8}{|c|}{ Porcentajes } \\
\hline & Media & $\begin{array}{l}\text { Medi } \\
\text { ana }\end{array}$ & $\begin{array}{l}\text { Desv. } \\
\text { Típica }\end{array}$ & $\begin{array}{l}\text { Nunc } \\
\text { a }\end{array}$ & $\begin{array}{l}\text { Casi } \\
\text { Nunca }\end{array}$ & $\begin{array}{l}\text { A } \\
\text { veces }\end{array}$ & $\begin{array}{l}\text { Casi } \\
\text { siempr } \\
\text { e }\end{array}$ & $\begin{array}{l}\text { Siempr } \\
\mathrm{e}\end{array}$ \\
\hline $\begin{array}{lr}\text { 17. Me intereso por } \\
\text { conocer } & \text { los } \\
\text { intereses } & \\
\text { profesionales } & \text { de } \\
\text { mis alumnos } & \end{array}$ & 4.65 & 5.0 & .606 & - & 1.75 & 1.7 & 26.7 & 70.0 \\
\hline $\begin{array}{l}\text { 18. Fomento la } \\
\text { reflexión y el } \\
\text { autoconocimiento } \\
\text { de mis alumnos para } \\
\text { favorecer la toma } \\
\text { de decisiones }\end{array}$ & 4.50 & 5.0 & .701 & - & 1.7 & 6.7 & 31.7 & 60.0 \\
\hline $\begin{array}{lr}\text { 19. Guío a mis } \\
\text { alumnos rara } \\
\text { fomentar } \\
\text { desarrollo personal } \\
\text { y profesional }\end{array}$ & 4.52 & 5.0 & .701 & - & 1.7 & 6.7 & 30.0 & 61.7 \\
\hline $\begin{array}{l}\text { 20. Asesoro a mis } \\
\text { alumnos en la } \\
\text { planificación de su } \\
\text { carrera personal }\end{array}$ & 4.47 & 5.0 & .747 & - & 1.7 & 10.0 & 28.3 & 60.0 \\
\hline $\begin{array}{l}\text { 21. Oriento a mis } \\
\text { alumnos en la } \\
\text { planificación de su } \\
\text { futura formación } \\
\text { permanente para el } \\
\text { desarrollo de su } \\
\text { profesión }\end{array}$ & 4.53 & 5.0 & .676 & - & 1.7 & 5.0 & 31.7 & 61.7 \\
\hline Total & 4.53 & 4.90 & .611 & & & & & \\
\hline
\end{tabular}

Objetivo 5: Conocer la satisfacción del profesorado de Formación Profesional respecto al ejercicio de la AT.

En la Tabla 6, podemos observar que las medias obtenidas del profesorado participante oscilan entre 3 y 4 , con una media global de 3.70 , de manera que podemos decir que esta dimensión obtiene unos resultados positivos.

En cuanto al deseo de mejorar la formación en materia de AT, los resultados son positivos ya que un $75 \%$ de los profesores encuestados indica que siempre o casi siempre le gustaría mejorar su preparación acerca de esta temática. Además, del análisis inferencial extraemos 
que los profesores que no son tutores tienen una disposición estadísticamente significativa más positiva a mejorar su formación en materia de AT, con una significación estadística de $p=.032$ y un tamaño del efecto $d$ de Cohen superior a .50 .

Tabla 6.

Estadísticos descriptivos de la dimensión Satisfacción

\begin{tabular}{|c|c|c|c|c|c|c|c|c|}
\hline \multicolumn{9}{|c|}{ Porcentajes } \\
\hline Ítem & Media & $\begin{array}{l}\text { Median } \\
\text { a }\end{array}$ & $\begin{array}{l}\text { Desv. } \\
\text { Típica }\end{array}$ & Nunca & $\begin{array}{l}\text { Casi } \\
\text { Nunca }\end{array}$ & A veces & $\begin{array}{l}\text { Casi } \\
\text { siempr } \\
\text { e }\end{array}$ & $\begin{array}{l}\text { Siempr } \\
\mathrm{e}\end{array}$ \\
\hline $\begin{array}{l}22 . \quad \text { Estoy } \\
\text { satisfecho con mi } \\
\text { labor en materia } \\
\text { de AT }\end{array}$ & 3.90 & 4.0 & .796 & - & 5.0 & 21.7 & 51.7 & 21.7 \\
\hline $\begin{array}{l}23 . \quad \mathrm{Mi} \\
\text { motivación en el } \\
\text { desempeño de la } \\
\text { AT es alta }\end{array}$ & 4.17 & 4.0 & .668 & - & - & 15.0 & 53.3 & 31.7 \\
\hline $\begin{array}{l}\text { 24. He recibido } \\
\text { formación sobre } \\
\text { AT }\end{array}$ & 3.13 & 3.0 & 1.268 & 15.0 & 10.0 & 40.0 & 16.7 & 18.3 \\
\hline $\begin{array}{l}\text { 25. Mi formación } \\
\text { inicial me ha } \\
\text { proporcionado } \\
\text { competencias } \\
\text { para desarrollar } \\
\text { la AT en el aula }\end{array}$ & 3.23 & 3.0 & 1.254 & 8.3 & 21.7 & 30.0 & 18.3 & 21.7 \\
\hline $\begin{array}{l}\text { 26. Me gustaría } \\
\text { mejorar mi } \\
\text { formación en } \\
\text { materia de AT }\end{array}$ & 4.05 & 4.0 & .872 & - & $5.0 \%$ & 20.0 & 40.0 & 35.0 \\
\hline Total & 3.70 & 3.60 & .715 & & & & & \\
\hline
\end{tabular}

\section{Discusión y conclusiones}

La AT está integrada en las aulas de Formación Profesional, pero los profesores dan respuestas negativas cuando se les pregunta si el tiempo dedicado a esta actividad es suficiente, coincidiendo con Álvarez y Álvarez (2015) donde afirman que entre las dificultades que encuentra el profesorado a la hora de integrar la AT en el aula está la falta de tiempo, y como se ha expuesto en el marco teórico la tutoría colectiva carece de hora lectiva dentro del horario de Formación Profesional, excepto en el módulo de Formación en Centros de trabajo y el módulo de proyecto. Esta circunstancia condiciona esa percepción acerca del escaso tiempo que el profesorado tiene, pues la AT se ha de llevar a cabo transversalmente en la acción docente diaria.

En base a los resultados podemos decir que respecto a la relación entre los docentes participantes y el Departamento de Orientación ésta es positiva, aunque con determinados aspectos que mejorar, por una parte, los docentes muestran cierta carencia en la demanda de asesoramiento y en su coordinación con este Departamento, atendiendo a las funciones 
del profesorado que establece la LOE (2006), y las funciones de los tutores que establece el ROI-ES.

Por otra parte, respecto a la relación de los docentes participantes con el Plan de Acción Tutorial, concluimos que también es adecuado, aunque podemos decir que la participación de los docentes en su desarrollo es baja teniendo en cuenta las funciones que establece el ROI-ES para los tutores. No obstante, debemos tener en cuenta que solo el $63 \%$ de los profesores encuestados ejerce como tutor de grupo, por lo que este resultado se ve atenuado y podemos decir que la relación de los docentes participantes respecto al PAT se encuentra en niveles aceptables.

En cuanto a las relaciones que se establecen entre los profesores, podemos decir que son positivas, ya que los docentes participantes consideran que la coordinación docente mejora su propia docencia, algo que concuerda con lo establecido por Álvarez (2017) y Torres (1996) que se refieren a la coordinación entre profesores como una relación positiva para la docencia en general y la AT en particular.

Cuando hablamos de las relaciones en el aula de Formación Profesional, podemos decir que la AT contribuye a mejorar tanto la relación profesor-alumno, como las relaciones con el centro y entre los propios alumnos, ya que los resultados obtenidos son muy positivos respecto a los ítems referidos a las relaciones en el aula. Este argumento lo vemos reforzado por Serrano (2009) y García et al. (2016), que se refieren a la tutoría como una acción que incide de manera positiva en el clima del aula y en la socialización del grupo. Otros autores apoyan estos resultados, tales como Castaño et al. (2012) que valoran el conocimiento individual de los alumnos para mejorar su desarrollo, y por Álvarez (2008) que concuerda en este aspecto y además cree que la tutoría es una oportunidad para profundizar en el conocimiento de cada uno de los alumnos.

En lo que se refiere a la implicación del profesorado de Formación Profesional en el desarrollo profesional del alumnado, los resultados obtenidos son positivos, tanto en la disposición de los profesores a conocer los intereses profesionales de sus alumnos, como en la orientación, asesoramiento y guía del futuro académico y profesional de los alumnos, lo que se ajusta a las funciones del profesorado según establece la LOE (2006) y las funciones del tutor según establece el ROI-ES, respecto a la orientación académica y profesional del alumnado.

El quinto objetivo de este trabajo consistía en conocer la satisfacción del profesorado de Formación Profesional respecto al ejercicio de la acción tutorial, en este punto concluimos que una gran parte del profesorado participante se encuentra satisfecho y motivado con respecto a su labor en AT y que, en general, muestra predisposición a seguir formándose para mejorar su docencia, algo que contrasta con Álvarez y Álvarez (2015) que señalan la falta de interés del profesorado como dificultad para el desarrollo de la AT en el aula. Por tanto, este escenario es bastante optimista respecto a la manifestación del alto interés por seguir recibiendo formación por parte del profesorado participante.

Respecto a la formación del profesorado en AT y su disposición a seguir formándose en esta materia, el porcentaje de profesores que ya ha recibido formación en AT es discreto, algo que concuerda con lo expuesto en el marco teórico, cuando hacemos referencia a que no a todos los profesores de Formación Profesional que se encuentran activos actualmente se les ha exigido una formación pedagógica inicial para acceder al ejercicio de la docencia según LOGSE (1990). Sin embargo, la disposición de los profesores a formarse es alta, y además son los profesores que no ejercen como tutores los que más interés muestran en su formación en AT, lo que nos indica que, aunque no ejerzan como tutores, le dan importancia a esta labor, lo que concuerda con Arnaiz y Riart (1999) y Álvarez (2017), que hablan de la necesidad de que cualquier profesor esté formado en AT. 
Por ello podemos concluir que los docentes de Formación Profesional participantes, en general, valoran la tutoría como un ejercicio beneficioso sobre las relaciones en el aula en esta etapa educativa, a la vez que consideran contribuye a mejorar diferentes aspectos en la evolución de los alumnos a nivel académico, profesional y personal.

La AT se lleva a cabo en las aulas de Formación Profesional, aunque los profesores participantes admiten que no siempre el tiempo destinado a esta labor es suficiente, por otra parte, no existe un horario lectivo para el desarrollo de esta actividad en el aula, excepto en el módulo de Formación en Centros de trabajo y en el módulo de Proyecto, ambos módulos se desarrollan en la fase final de la formación de los alumnos de Ciclos Formativos, lo que nos lleva a plantearnos, como propuesta, la asignación de un horario lectivo que dé cabida a la tutoría colectiva durante el resto del curso académico, para así conseguir una mejor integración de la AT en el aula.

\section{Referencias}

Álvarez, M. (2019), “Análisis de la percepción del profesorado sobre la función tutorial en ESO y bachillerato", ENSAYOS Revista de la Facultad de Educación de Albacete, vol. 34, núm. 2, pp. 19-34.

Álvarez, M. (2020) "Algunas valoraciones del profesorado respecto a su función tutorial”, Educatio Siglo XXI, vol. 38, núm. 1, pp. 139-160.

Álvarez, M. y Álvarez, J. (2015), "La tutoría universitaria: del modelo actual a un modelo integral”, Revista Electrónica Interuniversitaria de Formación del Profesorado, vol. 18, núm. 2, pp. 125-142.

Álvarez, M. (2017), "Hacia un modelo integrador de la tutoría en los diferentes niveles educativos", Revista Educatio Siglo XXI, vol. 35, núm. 2, pp. 21-42.

Álvarez, M. (2008), “La tutoría académica en el Espacio Europeo de la Educación Superior”, Revista Interuniversitaria de Formación del Profesorado, vol. 22, núm. 1, pp. 71-88.

Arnaiz, P. y Riart, J. (1999), La tutoría: de la reflexión a la práctica, Barcelona, Les Punxes Distribuidora, S.L.

Bisquerra, R. (ed.). (2009), Metodología de la investigación educativa, Madrid, La Muralla.

Caja Núñez, C., Cascales- Martínez, Antonia y Gomariz, M. A. (2017), "Valoración desde la perspectiva de los orientadores de Educación Secundaria de la Región de Murcia del portal de Atención a la Diversidad ORIENTAMUR", Revista Electrónica Interuniversitaria De Formación Del Profesorado, vol.20, núm. 1, pp. 49-68.

Castaño, E., Blanco, A. y Asensio, E. (2012), “Competencias para la tutoría: experiencia de formación con profesores universitarios", Revista de Docencia Universitaria, vol. 10, núm. 2, pp. 193-210.

Castillo, S., Torres, J. y Polanco, L. (2009), Tutoría en la enseñanza, la universidad y la empresa, Madrid, Pearson Educación, S.A.

Cerón Gómez, A., Cascales Martínez, A. y Gomariz Vicente, M. A. (2019), Cuestionario sobre el desarrollo de la acción tutorial en Ciclos Formativos, Murcia, Editum.

Cohen, J. (1988) ( $2^{\mathrm{a}}$. ed.), Statistical Power Analysis for the Behavioral Sciences, New York, Academic Press. 
Comunidad Autónoma Región de Murcia (2015), Resolución de 9 de abril de 2015, de la Dirección General de Formación Profesional por la que se dictan instrucciones para la puesta en marcha y desarrollo del módulo de Formación en Centros de Trabajo para los alumnos matriculados en centros docentes de la Comunidad Autónoma de Murcia, (Murcia, Consejería de Educación, Juventud y Deportes.

Comunidad Autónoma Región de Murcia (2015), "Reglamento Orgánico de Institutos de Educación Secundaria", Murcia.

Comunidad Autónoma Región de Murcia (2018), Resolución de 8 de noviembre de 2018 de la Dirección General de Formación Profesional y Enseñanzas de Régimen Especial por la que se dictan instrucciones para el desarrollo del módulo profesional de Proyecto de los ciclos formativos de Formación Profesional en el ámbito de la Comunidad Autónoma de la Región, (BORM nº 269 de 21 noviembre), Murcia, Consejería de Educación, Juventud y Deportes.

Delors, J., Informe a la UNESCO de la Comisión Internacional sobre la educación para el siglo XXI, presidida por JACQUES DELORS La Educación Encierra un Tesoro. 1996.

De Vellis, R. (2003) (2a. ed.), Scale development: Theory and applications, Thousand Oaks CA, Sage.

García, B., Ponce, S., García, M., Caso, J., Morales, C., Martínez, S. y Aceves, Y. (2016), “Las competencias del tutor universitario: una aproximación a su definición desde la perspectiva teórica y de la experiencia de sus actores", Perfiles Educativos, vol. 38, núm. 151, pp. 104-122.

González, M. (2018), “La función tutorial en Formación Profesional”, Espiral, vol. 11, núm. 23, pp. 130-140.

Lázaro, Á. (1997), "La AT de la función docente universitaria”, Revista complutense de educación, vol. 8, núm. 1, pp. 233-252.

Gobierno de España (1990), Ley Orgánica 1/2990, de 3 de octubre, General del Sistema Educativo (BOE $\mathrm{n}^{\circ} 238$, de 4 de octubre), Madrid, Ministerio de Educación y Ciencia.

Gobierno de España (1996). Real Decreto 83/1996, de 26 de enero, por el que se aprueba el Reglamento Orgánico de los Institutos de Educación Secundaria, (BOE nº 45, de 21 de febrero), Madrid, Ministerio de Educación y Ciencia.

Gobierno de España (2006), Ley Orgánica 2/2006, de 3 de mayo, de Educación (BOE nº 106, de 4 de mayo), Madrid, Ministerio de Educación y Ciencia.

Gobierno de España (2013), Ley Orgánica 8/2013, de 9 de diciembre, para la Mejora de la Calidad Educativa (BOE n 295, de 10 de diciembre), Madrid, Ministerio de Educación y Ciencia.

López Mayor, Cristian y Cascales Martínez, Antonia (2019), “Acción tutorial y tecnología: propuesta formativa en educación primaria", Revista Electrónica Interuniversitaria De Formación Del Profesorado, vol. 22, núm. 3, pp. 233-249.

Narro Robles, J. y Arredondo Galván, M. (2013), “La tutoría: un proceso fundamental en la formación de los estudiantes universitarios.", Perfiles educativos, vol. 35, núm. 141, pp. $132-151$.

Olmos Rueda, P. \& Mas Torelló, O. (2017), "Perspectiva de tutores y de empresas sobre el desarrollo de las competencias básicas de empleabilidad en el marco de los programas de formación profesional básica.", Educar, vol. 53, núm. 2, pp. 261-284. 
Sánchez, C., Campillo, S., De Nicolás, Jerónimo y Peral, C. (2016), Orientación y Acción Tutorial: Máster Universitario en Formación del Profesorado, Murcia, Servicio de Publicaciones de la Universidad de Murcia.

Serrano, C. (2009), "AT y clima escolar en los centros educativos a juicio del profesorado de Educación Secundaria Obligatoria", Puls, vol. 32, pp. 95-121.

Tárrida, A. (2012), “Perfil competencial del tutor o de la tutora de enseñanza secundaria”, REOP, vol. 23, núm. 2, pp. 22-41.

Torrego, J. C., Gómez, M., Martínez, C. y Negro, A. (2014), 8 Ideas clave: la tutoría en los centros educativos, Barcelona, Graó.

Torres, J. A. (1996), La formación del profesor tutor como orientador, Jaén, Publicaciones de la Universidad de Jaén. 